\title{
Rapid nodal staging of head and neck cancer surgical specimens with flow cytometric analysis
}

\author{
Valtteri Häyry ${ }^{\star 1,2}$, Åsa Kågedal ${ }^{1,2}$, Eric Hjalmarsson ${ }^{1,2}$, Pedro Farrajota Neves da Silva ${ }^{3}$, Cecilia Drakskog ${ }^{1,2}$, \\ Gregori Margolin ${ }^{1,2}$, Susanna Kumlien Georén ${ }^{1,2}$, Eva Munck-Wikland ${ }^{1,2}$, Ola Winqvist ${ }^{4}$ and \\ Lars Olaf Cardell ${ }^{1,2}$ \\ ${ }^{1}$ Division of ENT Diseases, Department of Clinical Sciences, Intervention and Technology, Karolinska Institutet, 17177 Stockholm, \\ Sweden; ${ }^{2}$ Department of Otorhinolaryngology, Karolinska University Hospital, 17177 Stockholm, Sweden; ${ }^{3}$ Department of \\ Pathology and Cytology, Karolinska University Hospital, 17164 Stockholm, Sweden and ${ }^{4}$ Department of Medicine, Unit of \\ Immunology and Allergy, Karolinska Institutet, 17176 Stockholm, Sweden
}

Background: Detection of metastatic spread of head and neck cancer to cervical lymph nodes is essential for optimal design of therapy. Undetected metastases lead to mortality, which can be prevented by better detection methods.

Methods: We analysed 41 lymph nodes from 19 patients with oral squamous cell carcinoma (OSCC). Each lymph node was divided in two, one half processed for histopathology and the other half dissociated into single-cell suspension, stained for the carcinoma cell markers cytokeratin 5/8 (CK5/8), epithelial cell adhesion molecule (EpCAM) and epithelial mucin (MUC-1), and analysed with flow cytometry. Flow cytometry data were compared with histopathology performed on serial sections and immunohistochemistry. Six cervical lymph nodes from cancer-free patients were used to establish baseline levels in flow cytometry.

Results: Flow cytometry analysis (fluorescence-activated cell sorting; FACS) detected all six metastases confirmed by histopathology as well as the histologically negative nodes. Importantly, among nine sentinel lymph nodes, FACS analysis detected $<1 \%$ malignant cells in four cases, not found in histopathology. Results from flow cytometry analysis can be obtained within $3 \mathrm{~h}$ of the time of biopsy.

Conclusions: We show that flow cytometric analysis of nodal tissue is sensitive and reliable in identifying metastases of OSCC. Flow cytometry is inexpensive and fast, providing a possibility of perioperative diagnostics and immediate treatment planning.

Accurate nodal staging is essential for successful treatment of oral cancer (oral squamous cell cancer (OSCC)). Metastatic spread to cervical lymph nodes is the most common reason for relapse or mortality. Because the rate of metastasis that is undetected by clinical investigation and imaging is $\sim 20 \%$ in stage T1 and T2 oral cancer, surgery is usually recommended (Psychogiros et al, 2013). Surgical neck dissection is used to evacuate lymph nodes at risk of metastasis, and also for staging purposes. Elective neck dissection is shown to improve survival compared with watchful waiting in early-stage oral cancer (D'Cruz et al., 2015). The current Union for International Cancer Control classification of malignant tumours (TNM) determines lymph node metastasis, including micrometastasis, as a deposit of tumour tissue $>0.2 \mathrm{~mm}$ in diameter (Brierley et al, 2009). Standard histopathological investigation of neck dissection specimens is done by microscopy of haematoxylin-eosin (HE)-stained sections of all lymph nodes, but it is seldom that

*Correspondence: Dr V Häyry; E-mail: Valtteri.Hayry@fimnet.fi

Received 2 April 2017; revised 12 October 2017; accepted 16 October 2017; published online 23 November 2017

(C) 2018 Cancer Research UK. All rights reserved 0007 - 0920/18 
more than a couple of sections are taken from each lymph node. The amount of lymph node metastases that are missed, that is, the false negative rate, has been investigated in several studies and is estimated to be $5-58 \%$ when sections have been re-evaluated by immunohistochemistry (Ferlito et al, 2008). Sentinel lymph node (SLN) biopsy with serial sectioning and immunohistochemistry has been studied as an alternative to neck dissection, and could detect metastatic spread with $\sim 90 \%$ accuracy and a negative predictive value of $96 \%$ (Civantos et al, 2010). It has also been shown that clinically node-positive, but histopathologically node-negative patients have a worse survival than clinically and histopathologically node-negative patients (Amit et al, 2013).

Diagnostic sensitivity can be improved by immunohistochemical staining for epithelial cell markers, although no OSCC-specific markers are known. The most commonly used marker is the pancytokeratin antibody AE1/AE3, which is shown to have a better sensitivity than antibodies recognising only cytokeratins of a particular molecular weight (Yamauchi et al, 2011). However, the AE1/AE3 antibody also recognises cytokeratin that is naturally present in normal epithelial tissue such as salivary glands. Epithelial cell adhesion molecule (EpCAM) is overexpressed in oral cancer, especially the invasive front, and downregulation of EpCAM in cell lines decreased invasiveness (Yanamoto et al, 2007). However, several independent studies have provided contradictory results regarding EpCAM expression and the metastatic potential of squamous cell carcinoma (SCC; reviewed by van der Gun et al (2010)). Epithelial mucin (MUC-1), a transmembrane glycoprotein, is another potential marker of OSCC, especially if the cytoplasmic portion of the molecule is probed (Rabassa et al, 2006).

Several experimental methods to detect tumour cells and metastases in lymph nodes have been described. The majority of these apply PCR or microarray techniques that target one or several tumour cell markers (reviewed by Ferlito et al (2008)). Quantitative RT-PCR could detect cytokeratin 5 and 14 in a majority of head and neck tumours, although the sample size was relatively small (Becker et al, 2004). In a large study of 1328 lymph nodes from 31 patients, targeting cytokeratin 19 mRNA with RTPCR could distinguish between metastatic and non-metastatic nodes, and detected cytokeratin 19 more often than immunohistochemistry (Tao et al, 2006). Although PCR-based methods are highly sensitive, the reported presence of mRNA for given markers does not necessarily correspond with protein expression detected by immunohistochemistry. Flow cytometry provides the benefit of being able to detect marker expression in intact cells as opposed to cell lysates, and therefore provides evidence of actual viable tumour cells in a sample. Flow cytometry has recently been demonstrated to be efficient in detecting micrometastases in renal cell carcinoma (Hartan et al, 2016).

In this proof-of-concept study, we investigate whether lymph node metastases of oral cancer can be detected using a rapid flow cytometry analysis. Flow cytometry is suitable for fast screening of a sample, and enables the analysis of the whole lymph node compared to histologically analysed tissue sections that represent only a small fraction of the total sample volume.

\section{MATERIALS AND METHODS}

Patients. Altogether, 19 patients with OSCC of the tongue, who were recruited at the ENT-HNS department of the Karolinska University Hospital, were included in this study. The study was approved by the local ethics committee (EPN-Stockholm) and all patients provided their written informed consent. All patients had been scheduled for primary surgical treatment, including neck dissection. One patient had received curative treatment for hypopharyngeal SCC 5 years earlier, while all other patients had not received previous chemo- or radiotherapy to the head and neck region. Tissue samples of the primary tumour and lymph nodes, including metastatic nodes, were collected at the time of primary surgical treatment. In six patients, SLNs were identified as follows: Technetium Tc 99m-labeled colloidal human serum albumin (Nanocoll, GE-Healthacare, Little Chalfont, UK) was injected around the tumour and lymphoscintigraphy was performed before surgery. Intraoperative patent blue injection to the primary site was done, and blue staining in addition to a handheld gamma probe was used to localise the sentinel nodes. Patient data are summarised in Table 1. Six normal lymph nodes from four cancer-free patients were collected during surgery of the neck for benign causes. These samples were treated in an identical manner as the above cancer patient samples.

Tissue sample processing. Samples for this study were collected during surgery. Following resection, neck dissection and tongue specimens were kept at $4{ }^{\circ} \mathrm{C}$. Clinically evident lymph node metastases and non-metastatic lymph nodes from a neck level proximal (levels I-II) and distal (levels III-V) to the tongue were identified. Sentinel lymph nodes had been marked intraoperatively. Lymph nodes were divided and one-half of each lymph node was collected for the study; the remaining half was submitted for routine clinical pathology. Samples were taken from each SLN or metastasis (if available), as well as a clinically non-metastatic node for comparison. In such cases where no metastatic node or SLN was present, a lymph node proximal to the tongue was chosen. Also, a sample from the primary tumour of the tongue was taken from all except four cases, in which representative tissue was scarce. Collected samples were placed in tissue storage solution (Miltenyi Biotec, Cologne, Germany) and kept at $4{ }^{\circ} \mathrm{C}$.

Flow cytometry (fluorescence-activated cell sorting). Before carrying out the current experiments, we determined optimal tissue processing and antibody staining conditions using the head and neck SCC cell line, FaDu (American Type Culture Collection, Manassa, VA, USA), and samples of primary SCC tumour tissue and lymph nodes from additional OSCC patients not included in this study (data not shown). Primary human nasal airway epithelial cells (obtained by nasal brushing, as previously described in Rydberg et al (2009)), human bronchial airway smooth muscle cells (Promocell, Heidelberg, Germany) and tongue tissue (obtained from tumour resection specimens) were used as additional controls (data not shown). The panel of markers and corresponding flow-cytometry antibodies used in this study was also optimised in beforehand. Interestingly, we tested several commercially available fluorescence-activated cell sorting (FACS)antibodies representing the anti-pan-cytokeratin clone AE1/AE3, all of which demonstrated significant background signal levels when lymph nodes were analysed with FACS. Therefore, we screened several other antibodies recognising specific cytokeratins of a particular molecular weight, and found the anti-cytokeratin 5/ 8 antibody (described in detail below) to be most suitable for this study. Isotype and FMO controls were used in antibody stainings.

Flow cytometry was usually performed the same day as surgery, or latest within $24 \mathrm{~h}$ after surgery. Optimally, the whole procedure can be performed in $<3 \mathrm{~h}$. Tissue samples were dissociated using a 'Gentle MACS' apparatus and human tumour dissociation kit using the 'tough tumour' protocol provided by the manufacturer (Miltenyi Biotec). Samples were flushed through a $100 \mu \mathrm{m}$ cell strainer, centrifuged, and washed with phosphate-buffered saline.

Antibody staining was performed using the Perfix-nc intra- and extracellular staining preparation kit (Beckman Coulter, Brea, CA, USA). In brief, dissociated tissue samples were suspended in BV Brilliant Stain Buffer (BD Biosciences, San Jose, CA, USA), incubated with fixation reagent for $15 \mathrm{~min}$, then incubated for 10 min with permeabilisation reagent supplemented with 1:50 Human Fc block (BD Biosciences). Antibodies were then added to 
Table 1. Clinical data of patients with tumour in the oral tongue

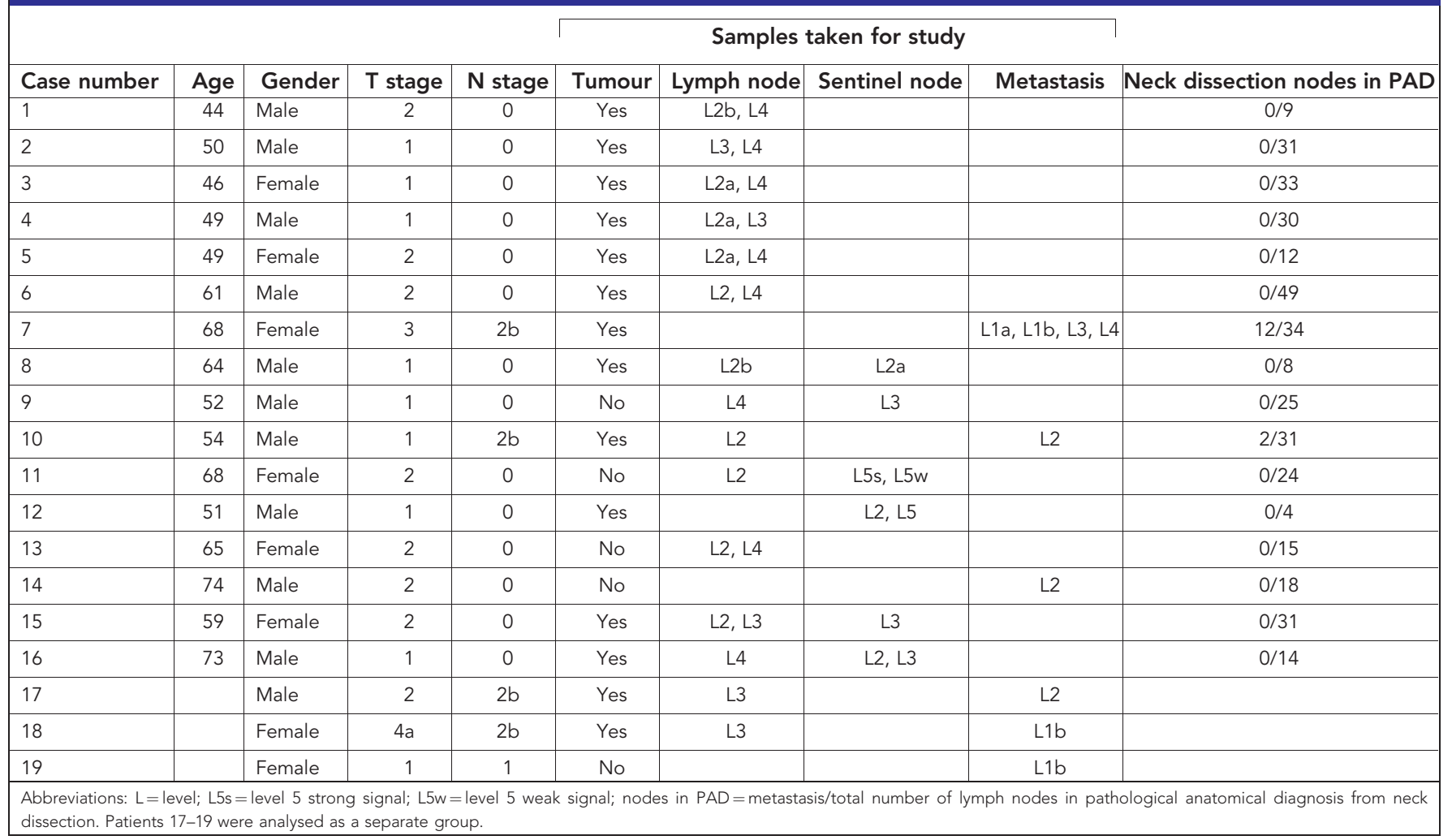

the samples and incubated for $15 \mathrm{~min}$; samples were finally washed twice with washing reagent.

The antibodies used were anti-human cytokeratin 5/8 (1:100 dilution, clone RCK102, Novus Biologicals, Littleton, CO, USA), EpCAM (1:25 dilution, clone EBA-1, BD Biosciences) and mucin 1 (MUC-1) (1:50 dilution, clone HMPV, BD Biosciences). All antibodies were fluorescein isothiocyanate (FITC)-conjugated. The FITC fluorescent signal, obtained from each of the antibodies separately, and all three in combination are demonstrated in Figure 1A. An example of a tumour cell stained with all three antibodies is presented in Figure 1B. We observed frequent FITCpositive fragments or debris, especially in metastasis and primary tumour samples. These particles were not true cancer cells as they lacked a cell nucleus, possibly representing phagocytosed material or necrotic cell fragments (Figure 1C). To decrease false-positive signals and ensure that only viable, intact tumour cells were measured, we added a nuclear stain of propidium iodide to our protocol (Figure 1D). Flow cytometry was performed on a BD Accuri cytometer (BD Biosciences) and FACS data were analysed using FloJo software (Tree Star, Ashland, OR, USA). The analysis strategy (population gating) is presented in Figure 1C-E.

Histopathology and immunohistochemistry. Half of each lymph node specimen was submitted to routine clinical pathology examination according to the routine guidelines of our institution. Specifically, 1-3 sections from the middle of each lymph node were stained with HE and screened for the presence of metastases in light microscopy by a specialist in head and neck pathology (PFNdS). Sentinel lymph nodes were step-serial sectioned in three levels with $50 \mu \mathrm{m}$ intervals and stained both with $\mathrm{HE}$ and immunohistochemistry with a pan-cytokeratin antibody.

We performed additional histological staining and immunohistochemistry on all lymph nodes included in this study to rule out false-negative results of standard clinical pathology examination.
All non-metastatic lymph nodes (N0) analysed by FACS were identified and the corresponding formalin-fixed, paraffinembedded fractions of these nodes were step-serial sectioned with $180 \mu \mathrm{m}$ between levels. Each level was stained with HE and two levels per node were also immunohistochemically stained with a pan-cytokeratin antibody (1:60 dilution, clones AE1/AE3, DAKO, Copenhagen, Denmark) as described previously (Millrud et al. 2013).

Statistics. Statistical analyses were done with GraphPad Prism version 6.01 (GraphPad Software, La Jolla, CA, USA). The D'Agostino-Pearson normality test was used to determine whether data sets were normally distributed, and one-way ANOVA or the Kruskall-Wallis tests were chosen, depending on the distribution of the data. The Brown-Forsythe test was conducted to evaluate homoscedasticity. Receiver-operating characteristic curves (ROCs) were plotted to calculate the area under the curve and evaluate sensitivity and specificity.

\section{RESULTS}

Metastatic deposits in lymph nodes can be detected with flow cytometry using a combination of epithelial cell markers. Cytokeratin 5/8, MUC-1, and EpCAM are epitopes common to epithelial cells and SCC. A combination of all three markers yields a robust signal in flow cytometry that can help identify tumour cells in OSCC samples. When a tumour sample was stained separately for each marker, the mean fluorescence intensity was 130895 units for EpCAM, 228333 units for MUC-1, and 307136 units for $\mathrm{CK} 5 / 8$, and for all three combined, 838000 units. Placing all markers under the same fluorescence channel therefore provides a good distinction between positive and negative events in flow cytometry. 
A

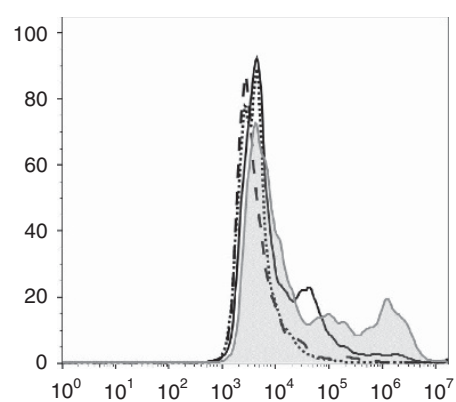

D

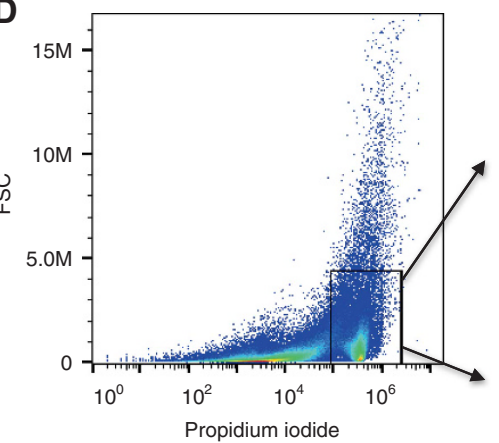

B

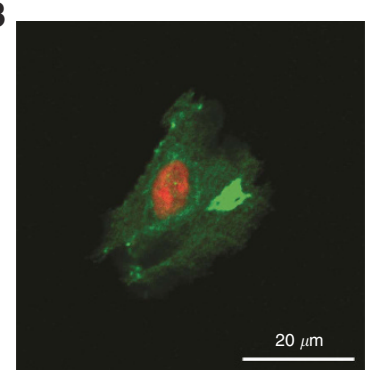

E

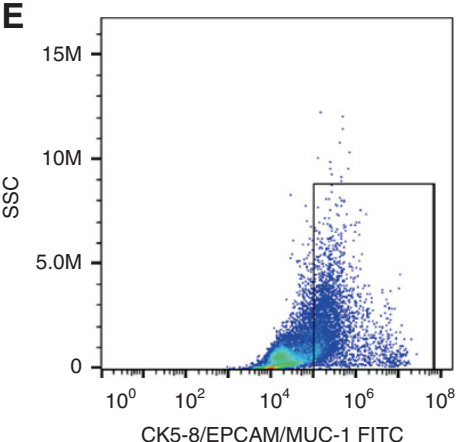

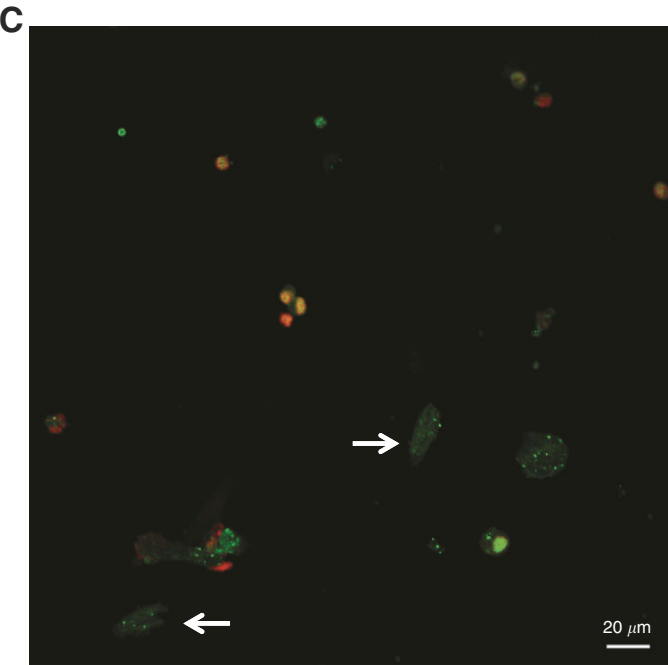

Figure 1. Details of flow cytometry method. (A) Histogram of FITC signal fluorescence from a tumour sample stained either with anti-CK5/8 (solid black line), anti-EpCAM (dotted line), or anti-MUC-1 (dashed line), and all three markers stained together (solid grey line). (B) A photomicrograph of a tumour cell, triple-stained with CK5/8, EpCAM, and MUC-1 FITC-conjugated antibodies (green) and propidium iodide (PI; red). (C) Several FITCpositive particles without a cell nucleus is seen among FITC-positive nucleated cells. Applying a nuclear stain helps to distinguish tumour cells from debris. (D, E) The gating strategy applied in FACS data analysis: first, PI-positive cells, that is, nucleated cells, were gated and from this subpopulation, the frequency of FITC-positive cells was quantified. (D) A PI gate, (E) a tumour sample with FITC-positive cells. CK5/8: cytokeratin 5/8.

All tissue samples were analysed by flow cytometry and the rate of epithelial marker positive cells were recorded. Retrospectively, the histopathological diagnosis was obtained and samples were grouped as primary tumour, metastasis, or non-metastatic lymph node. Sentinel lymph nodes and healthy control lymph nodes were grouped separately. Examples of flow cytometry results are shown in Figure 2.

We reasoned that because cutoff levels determining positive $v s$ negative signals (i.e., populations) in flow cytometry data can be determined subjectively, a sample can be diagnosed as positive for metastasis either based on an universally applied signal strength, that is, FITC fluorescence $>10^{5}$ units, or an individually determined signal strength based on the background (negative) level of each sample, that is (geometric mean fluorescence) + $(\mathrm{SD} \times 12)$. Therefore, we analysed data from all samples using both of the aforementioned parameters and found out that the relative amount (\%) of positive tumour cells in a sample is essentially the same regardless of the cutoff level applied. Results of all samples measured according to a fixed cut-off level are presented in Figure $3 \mathrm{~A}$, and the same samples measured according to individual cutoff levels are shown in Figure 3B. The Spearmann correlation between these two analysis strategies was $r=0.729, P<0.001$ and the non-linear regression model is presented in Figure 3C. Therefore, we can conclude that flow cytometry samples can reliably be analysed using a predetermined cutoff level for positive metastasis, meaning that the flow cytometry-based lymph node staging can be automatised.

In lymph nodes from cancer-free control patients, cells positive for EpCAM, CK5/8, and MUC-1 are found at a consistently low rate of $0.02-0.24 \%$ (median $0.21 \%$ ). Thus, the minimum level of detection for a positive sample is $0.35 \%$ positive cells per sample (mean (rate of positive cells in control samples) +2 SD (control)).
Using the analysis parameters described above, with a fixed (universal) cutoff level, the median rate of EpCAM/CK5/8/MUC1-positive cells in each sample was calculated (Figure $3 \mathrm{~A}$ ). The median rate of positive cells was $8.10 \%$ (range $2.46-33.00 \%$ ) in oral tumours and $3.9 \%$ (range 1.95-7.02\%) in histologically confirmed metastatic lymph nodes. The median rate of positive cells in histologically non-metastatic lymph node samples (N0) was $0.09 \%$ (range 0.02-1.04). Metastatic lymph nodes could therefore be reliably distinguished from non-metastatic nodes ( $P<0.0001$, Kruskal-Wallis test; $P<0.0001$, Mann-Whitney test). The median rate of positive cells in SLNs was 0.17 (range 0.0381.26) and did not differ from N0 nodes ( $P=$ NS, Mann-Whitney test).

Sensitivity of flow cytometric detection of metastatic cells. To confirm the true negative status of lymph nodes diagnosed as N0 in FACS analysis, additional histopathology was performed. The corresponding paraffin-embedded half of all N0 lymph node samples, excluding sentinel nodes, were serially sectioned entirely. Haematoxylin-eosin-stained sections of all levels were examined by an experienced pathologist. In addition, at least two pancytokeratin-stained sections per lymph node were examined. No false-negative FACS samples were found.

As seen in Figure 3A, three histologically N0 lymph nodes were EpCAM/CK5/8/MUC-1-positive in FACS analysis. However, no isolated tumour cells, micrometastases, or metastases were found in these nodes in histopathology. It is noteworthy that according to the current TNM classification (Brierley et al. 2009) small deposits of tumour tissue $(<0.2 \mathrm{~mm})$ and isolated tumour cells are classified as N0. Because the histopathological analysis of these FACS-positive nodes did not detect tumour cells, we cannot conclude whether our FACS result represents isolated tumour cells 

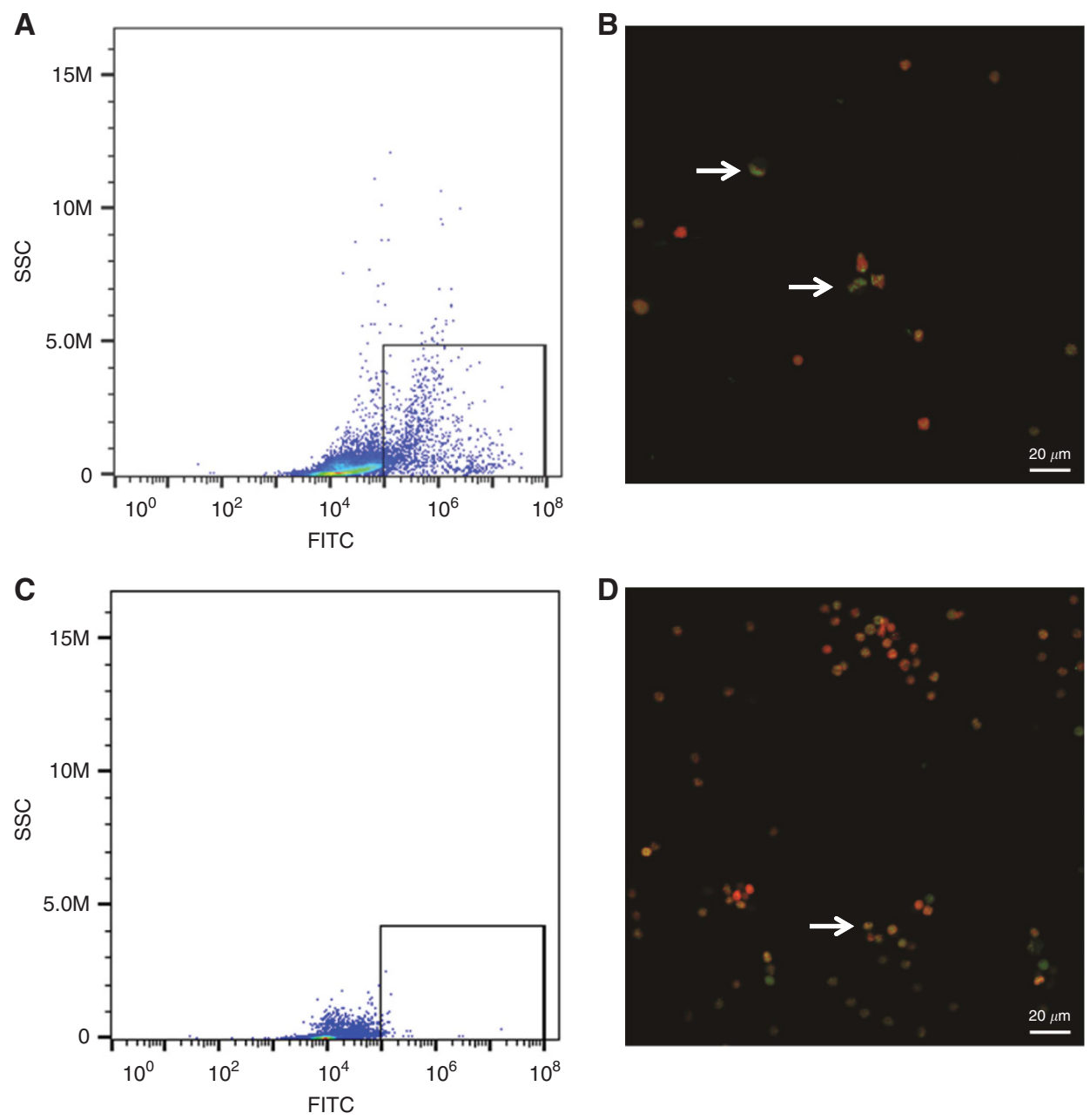

Figure 2. Tumour cells detected in lymph nodes. (A, B) Flow cytometry plot and photomicrograph of a metastatic lymph node sample with FITCpositive epithelial cells (green) amidst PI-positive (red) cells, which are mostly lymphocytes. (C, D) Flow cytometry plot and photomicrograph of a histopathologically non-metastatic SLN sample with isolated FITC-positive cells (green).

or micrometastases, but it suggests histopathology has a falsenegative rate of $15 \%$ in our material.

Importantly, 4 out of 9 SLNs were EpCAM/CK5/8/MUC-1positive in FACS analysis. The corresponding paraffin-embedded half of these nodes was analysed according to the standard method for SLNs with serial sections and pan-cytokeratin immunohistochemistry. All nine sentinel nodes were diagnosed as N0 and no isolated tumour cells were seen in light microscopy. The nodes positive in FACS analysis contained $\sim 1 \%$ EpCAM/CK5/8/MUC1-positive cells.

As seen in Figure 3A, the variance in the rate of tumour markerpositive cells in N0 nodes and in SLNs is larger than in healthy control lymph nodes (F-test, $P=0.0055$ and 0.0035 , respectively), suggesting that our finding of EpCAM/Ck8/MUC-1-positive cells in SLN and N0 nodes is specific as no positivity above the detection limit was observed among the healthy person-derived nodes.

We evaluated the sensitivity of the FACS method to distinguish between histopathologically metastatic and non-metastatic lymph nodes (including SLNs). In a ROC curve, the area under the curve was $1.000(P=0.00014)$, confirming excellent sensitivity $(100 \%)$.

Metastatic nodes can be detected with different types of flow cytometry systems. Finally, we tested whether or not our method of tumour cell detection could be applied to another type of flow cytometer, and analysed a set of samples from three separate patients using a BD LSRFortessa flow cytometer (Figure 3D).
When the same staining and processing parameters were applied, and gating strategy was adjusted according to the data output of the flow cytometer, similar results were obtained as when using the BD Accuri flow cytometer.

\section{DISCUSSION}

In this proof-of-concept study, we have applied flow cytometry to detect lymph node metastasis of OSCC. On the basis of academic database searches performed during the final stages of this study, no previous work of this kind in head and neck cancer has been published. Our data demonstrates that metastatic lymph nodes can be reliably distinguished from non-metastatic nodes. We could establish that all metastatic lymph nodes contained at least $1.95 \%$ tumour marker-positive cells, whereas the maximum amount of positive cells was $1.04 \%$ and $1.26 \%$ in N0 and SLNs, respectively. All SLNs included in this study were histopathologically negative for metastases, although in FACS analysis, 4 out of 9 SLNs were above the limit of detection. In other N0 nodes of the OSCC patients, 3 out of 20 were above the limit. This suggests that FACS has the ability to detect isolated tumour cells and micrometastases in SLNs with a sensitivity that is superior to standard pathology, although validation in a larger material is still needed. The volume of a lymph node has been estimated as $\sim 250 \mathrm{~mm}^{3}$; a metastasis represents $>1 \%$ of a lymph node's volume, and a micrometastasis 
A

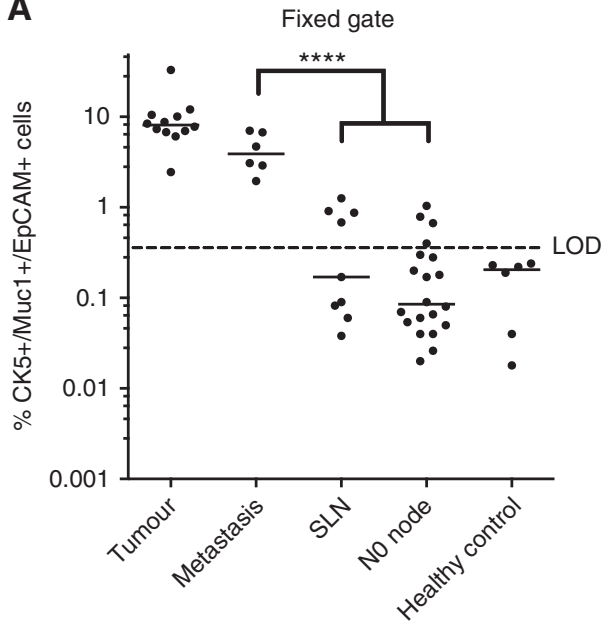

C

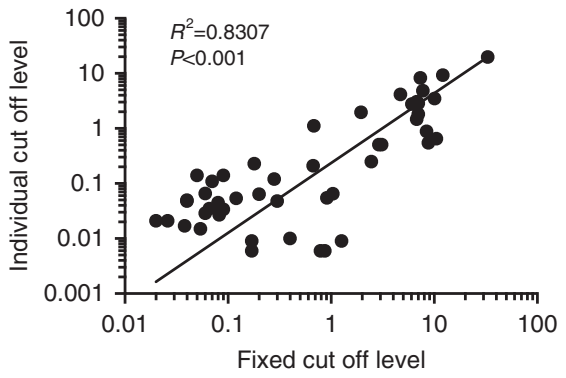

B

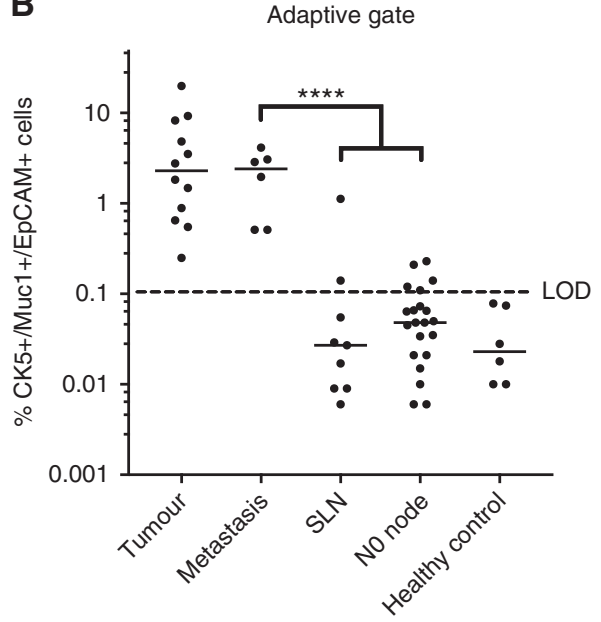

D

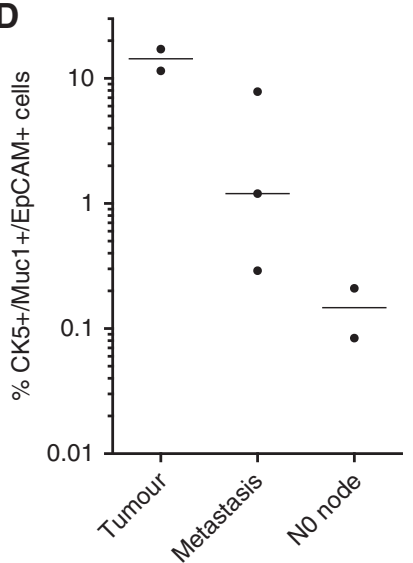

Figure 3. Summary of flow cytometry results. (A) the frequency of epithelial-marker-positive cells in primary tumour samples, metastases, histologically non-metastatic nodes (N0 node), control lymph nodes from cancer-free individuals, and SLNs. These samples were analysed with the BD Accuri flow cytometer and a fixed, universal cutoff level was applied. (B) The same samples as seen in A, now evaluated according to individually determined cutoff levels for each sample. (C) Non-linear regression between the signal strength cutoff levels applied in $\mathbf{A}$ and $\mathbf{B}$. (D) A separate set of samples, processed identically as the ones shown in $\mathbf{A}$ and $\mathbf{B}$, but analysed with a BD LSRFortessa flow cytometer. Note that the $y$ axes are logarithmic in $\mathbf{A}$ and $\mathbf{B}$, and both $x$ - and $y$-axes are logarithmic in $\mathbf{C}$. N0: histologically non-metastatic lymph node. ${ }^{\star \star \star \star} P$-value $<0.0001$.

0.032-1.6\% (Hartana et al, 2016). Therefore, analysing $>50 \%$ of a lymph node sample with flow cytometry enables precise nodal staging, including micrometastases.

There are no known markers specific for OSCC that are unambiguously expressed in every tumour of this type. Furthermore, most markers found in OSCC are also expressed in normal tissue of the oral cavity to varying degrees. Cervical lymph nodes, however, consist of lymphocytes, other leukocytes, dendritic cells, connective tissue, and endothelium, and normally should not contain epithelial tissue; thus, they should also be negative for epithelial markers. There are certain rare exceptions to this principle, notably epithelial inclusions, primarily from thyroid tissue, and also branchial arch remnants (Triantafyllou et al, (2016)). Expression of cytokeratins has been described in a small population of interstitial reticular cells of normal lymph nodes (Gould et al, 1995). In addition, during the process of metastasis, carcinoma cells undergo epithelial-to-mesenchymal transition and might lose expression of epithelial markers such as E-cadherin (Vig et al, 2015). Taken together, the choice of markers used to detect metastatic carcinoma cells is a balance between high sensitivity and an increase in false-positive findings. We chose three wellestablished markers of OSCC and defined all cells yielding a positive signal for any or all of these as putative metastatic cancer cells. This approach provides the flow cytometry method with certain robustness, as we found lymph nodes from healthy controls to be consistently more than $99.76 \%$ negative for these markers. Thus, any lymph node containing significantly more epithelial marker positive cells was considered to be aberrant, that is, metastatic.

In our method, all three antibodies were conjugated to the green fluorescent proteins FITC or AlexaFluor488. This approach has several advantages. As all three markers are used to detect the same cell population, it is sufficient if a cell is positive for one, but 'dim' for another marker, which makes data easy to interpret. Also, raw data analysis is simplified because multiple, possibly overlapping, threshold levels for individual markers are not needed. Other channels on the flow cytometer are left free, potentially allowing the simultaneous use of additional markers, for example, antibodies against p16 as a surrogate marker of human papilloma virus infection and epidermal growth factor receptor, both important clinical markers that influence the choice of therapy.

Elective neck dissection is the best treatment option in OSCC and is usually performed simultaneously as surgical resection of the primary tumour from the oral cavity. This type of surgery typically takes several hours to perform, and the possibility of obtaining intraoperative information of nodal metastases would be highly beneficial, enabling the surgical team to modify the extent of resection. In our current study, we could obtain a diagnosis using 
FACS within $3 \mathrm{~h}$ from biopsy, despite the fact that all laboratory work was done manually in separate buildings. It is feasible to shorten the time with semi-automatic laboratory equipment located 'bedside'.

Clinical and imaging methods evidently do not provide sufficient certainty to rule out nodal spread, because recurrent disease occurs much more often in patients not treated with primary node evacuation even with cN0 node status (D'Cruz et al, (2015)). In this kind of setting, accurate detection of metastases, for example, with FACS, prevents mortality.

In summary, this study provides proof of concept that flow cytometry can be applied in neck dissection staging in OSCC. In a clinical setting, where the need for standardised, automated diagnostic procedures and capacity to diagnose large numbers of samples rapidly is constantly increasing, flow cytometry provides the benefits of being fast, accurate, and objective. Furthermore, such automated procedures, easily performed by a technician, may eventually replace subjective microscopy of sectioned tissue samples in the diagnostic process.

\section{ACKNOWLEDGEMENTS}

We thank PhD Nele de Klerk for her superb technical assistance in performing antibody stainings and Dr Diana Shy for her editorial support of this manuscript. We also thank Sigrid Juseliuksen säätiö, Instrumentariumin tiedesäätiö, Suomen lääketieteen säätiö, and Finska Läkaresällskapet for providing financial support to Dr Häyry.

\section{CONFLICT OF INTEREST}

The authors declare no conflict of interest.

\section{REFERENCES}

Amit M, Yen TC, Liao CT, Binenbaum Y, Chaturvedi P, Agarwal JP, Kowalski LP, Ebrahimi A, Clark JR, Cernea CR, Brandao SJ, Kreppel M, Zöller J, Fliss D, Bachar G, Shpitzer T, Bolzoni VA, Patel PR, Jonnalagadda S, Robbins KT, Shah JP, Patel SG, Gil Z, International Consortium for Outcome Research (ICOR) in Head and Neck Cancer (2013) Clinical nodal stage is a significant predictor of outcome in patients with oral cavity squamous cell carcinoma and pathologically negative neck metastases: results of the international consortium for outcome research. Ann Surg Oncol 20: 3575-35781.

Becker MT, Shores CG, Yu KK, Yarbrough WG (2004) Molecular assay to detect metastatic head and neck squamous cell carcinoma. Arch Otolaryngol Head Neck Surg 130: 21-27.

Brierley JD, Gospodarowicz MK, Wittekind C (2009) TNM Classification of Malignant Tumours. 7th edn, Wiley-Blackwell: NJ, USA.

Civantos FJ, Zitsch RP, Schuller DE, Agrawal A, Smith RB, Nason R, Petruzelli G, Gourin CG, Wong RJ, Ferris RL, El Naggar A, Ridge JA, Paniello RC, Owzar K, McCall L, Chepeha DB, Yarbrough WG, Myers JN (2010) Sentinel lymph node biopsy accurately stages the regional lymph nodes for T1-T2 oral squamous cell carcinomas: results of a prospective multiinstitutional trial. J Clin Oncol 28: 1395-1400.
D’Cruz AK, Vaish R, Kapre N, Dandekar M, Gupta S, Hawaldar R, Agarwal JP, Pantvaidya G, Chaukar D, Deshmukh A, Kane S, Arya S, Ghosh-Laskar S, Chaturvedi P, Pai P, Nair S, Nair D, Badwe R, Head and Neck Disease Management Group (2015) Elective versus therapeutic neck dissection in node-negative oral cancer. N Engl J Med 373: 521-529.

Ferlito A, Rinaldo A, Devaney KO, Nakashiro K, Hamakawa H (2008) Detection of lymph node micrometastases in patients with squamous carcinoma of the head and neck. Eur Arch Otorhinolaryngol 265: $1147-1153$.

Gould VE, Bloom KJ, Franke WW, Warren WH, Moll R (1995) Increased numbers of cytokeratin-positive interstitial reticulum cells (CIRC) in reactive, inflammatory and neoplastic lymphadenopathies: hyperplasia or induced expression? Virchows Arch 425: 617-629.

van der Gun BT, Melchers LJ, Ruiters MH, de Leij LF, McLaughlin PM, Rots MG (2010) EpCAM in carcinogenesis: the good, the bad or the ugly. Carcinogenesis 31: 1913-1921.

Hartana CA, Kinn J, Rosenblatt R, Anania S, Alamdari F, Glise H, Sherif A, Winqvist $\mathrm{O}$ (2016) Detection of micrometastases by flow cytometry in sentinel lymph nodes from patients with renal tumours. Br J Cancer 115: 957-966.

Millrud CR, Kvarnhammar AM, Tajti J, Munck-Wikland E, Uddman R, Cardell LO (2013) Nod-like receptors in head and neck squamous cell carcinoma. Acta Otolaryngol 133: 1333-1344.

Psychogios G, Mantsopoulos K, Bohr C, Koch M, Zenk J, Iro H (2013) Incidence of occult cervical metastasis in head and neck carcinomas: development over time. J Surg Oncol 107: 384-387.

Rabassa ME, Croce MV, Pereyra A, Segal-Eiras A (2006) MUC1 expression and anti-MUC1 serum immune response in head and neck squamous cell carcinoma (HNSCC): a multivariate analysis. BMC Cancer 6: 253.

Rydberg C, Månsson A, Uddman R, Riesbeck K, Cardell LO (2009) Toll-like receptor agonists induce inflammation and cell death in a model of head and neck squamous cell carcinomas. Immunologyl 128(1 Suppl): e600e611.

Tao L, Lefèvre M, Ricci S, Saintigny P, Callard P, Périé S, Lacave R, Bernaudin JF, Lacau, Guily J (2006) Detection of occult carcinomatous diffusion in lymph nodes from head and neck squamous cell carcinoma using real-time RT-PCR detection of cytokeratin 19 mRNA. Br J Cancer 94: 1164-1169.

Triantafyllou A, Williams MD, Angelos P, Shah JP, Westra WH, Hunt JL, Devaney KO, Rinaldo A, Slootweg PJ, Gnepp DR, Silver C, Ferlito A (2016) Incidental findings of thyroid tissue in cervical lymph nodes: old controversy not yet resolved? Eur Arch Otorhinolaryngol 273: $2867-2875$.

Vig N, Mackenzie IC, Biddle A (2015) Phenotypic plasticity and epithelial-tomesenchymal transition in the behaviour and therapeutic response of oral squamous cell carcinoma. J Oral Pathol Med 44: 649-655.

Yamauchi K, Fujioka Y, Kogashiwa Y, Kohno N (2011) Quantitative expression study of four cytokeratins and p63 in squamous cell carcinoma of the tongue: suitability for sentinel node navigation surgery using onestep nucleic acid amplification. J Clin Pathol 64: 875-879.

Yanamoto S, Kawasaki G, Yoshitomi I, Iwamoto T, Hirata K, Mizuno A (2007) Clinicopathologic significance of EpCAM expression in squamous cell carcinoma of the tongue and its possibility as a potential target for tongue cancer gene therapy. Oral Oncol 43: 869-877.

This work is published under the standard license to publish agreement. After 12 months the work will become freely available and the license terms will switch to a Creative Commons AttributionNonCommercial-Share Alike 4.0 Unported License. 